\title{
Design of the New Wideband Circular Dipole Antenna
}

\author{
Bin $\operatorname{Lin}^{1}$, Xian Chen ${ }^{1, a}$, Hongjian Lin ${ }^{1}$, Jiawei Zheng ${ }^{1}$, Zijian Chen ${ }^{1}$, Zijian Lin $^{1}$ \\ ${ }^{1}$ Xiamen University Tan Kah Kee College, Fujian Zhangzhou 363105, China \\ aemail: 419425292@qq.com
}

Keywords: Wideband Antenna; Microstrip Antenna; Circular Fractal Antenna; Cage Dipole Antenna

\begin{abstract}
Basing on the operating wideband requirement for today's mobile communication environment, a new antenna named 'wide band circular dipole antenna', which has the advantages of wide band antenna, circular fractal antenna and cage dipole antenna, is designed. A sample of this antenna has been tested after simulation analysis. The result of simulation and test indicate that the antenna can fully covered the communication frequency of $1880-1920 \mathrm{MHz}, 2010-2025 \mathrm{MHz}$ of the third generation mobile communication TD-SCDMA system and also the communication frequency of $1710-1785 \mathrm{MHz}, 1880-1920 \mathrm{MHz}$ of the fourth mobile communication FDD-LTE system, in another hand, realize the compatibility of the third and the fourth generation mobile communication.
\end{abstract}

\section{Introduction}

As the information transformation becomes more portable today, people are more likely intend to use mobile communication device to improve life quality and work efficiency. The convenience comes with the mobile communication which makes people's life rich and colorful. Mobile interconnection is gradually becomes the majority way for worldwide information exchange and many kinds of mobile communication technology simultaneously living and developing on the world. It is predictable that in the future, different type of communication network system would coexist and being operated in different frequency.

Antennas, the most important role in wireless system, however, should have wider operating band to satisfy multiple systems operating condition. In the condition of rapid increasing demand for communication service, people are not only satisfied with stable communication quality, but also ask for the diversity of services. The extension of channels and the acceleration of information transformation also force the communication devices to have the characters of broadband. As antenna is the core part of mobile communication system, the meaning of its broadband design is significant [1-2].

Currently, for the third generation mobile communication system - TD-SCDMA, the majority frequency is 1880-1920 MHz and 2010-2025 MHz, while for the fourth generation mobile communication system - FDD-LET, the majority frequency is $1710-1785 \mathrm{MHz}$ and $1805-1880$ $\mathrm{MHz}$, and this requires the wide band antenna to have the coverage of four or more frequency ranges[3-4].

\section{The Application of Fractal Structure in Antenna Design}

In the process of designing antenna unit or antenna array, generally, applying fractal structure to antennas is an effective way to improve its performance. Comparing to the traditional antennas, fractal antenna has some unique advantages: Self-similarity is a symbolic feature of fractal structure, it can facilitate the extension of working frequency band. Moreover, applying fractal structure in antenna design can also stretch the electrical length in the squeezing process, keep the performance while maintaining its miniaturization. 'Self-loading' feature of fractal antenna can simplify the circuit and reduce cost.

Koch Curve, Hilbert Curve, Weierstrass Curve, Peano-Gosper Curve, Cantor Patch, Minkowski Patch, Sierpinski Carpet and Mandelbrot Tree are the main fractal structures mostly used in the 
design of antenna, of which the fractal curves are most commonly used [5-6]. Just like the meandering plank roads for sightseeing built from the straight roads, the application of such fractal curves in the design of antenna is conducive to reducing the size. In other words, the reconstruction of the road increases the distance with a magnitude of tens of times or even hundreds of times, namely increasing the length of curve. The radiation impedance of antenna increases as the length of curve and the decrease of $\mathrm{Q}$ factor which means its radiation is improved.

\section{Brief Introduction of Cage Antenna}

Shown as Figure 1, cage antenna is a kind of wideband dipole antenna, transformed from conventional dipole antenna. Conventional dipole antenna has wider directivity bandwidth and narrow impedance bandwidth because it has higher characteristic impedance and input impedance change greatly in the operational frequency band. Currently, the most of designing method of wideband dipole antenna is increasing the diameter of dipole arm to decrease the characteristic impedance of antenna and dipole arm is designed as cage type by using skin-effect of radiofrequency current [7].

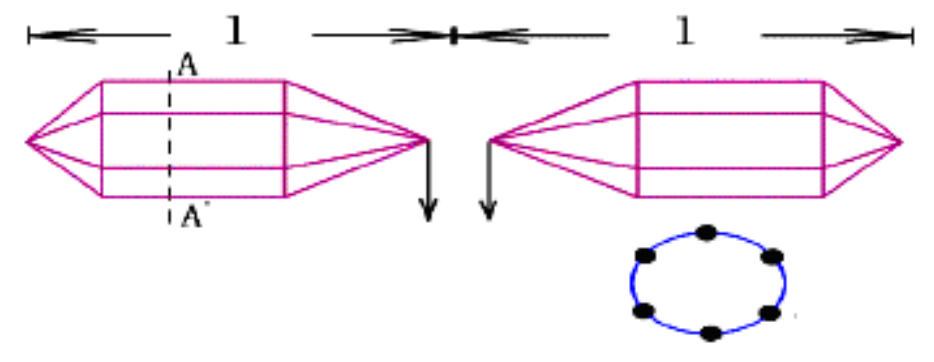

Fig.1. The scheme of current cage dipole antenna (The cross-section is regular hexagon)

Current cage dipole antennas with all metal structure and big size can't be put into mobile communication terminals. In order to apply cage dipole antenna to mobile communication, we should attempt to miniaturize it.

\section{The Design of Wideband Circular Dipole Antenna}

The aim of this article is to design wide band mobile communication antenna, which is compatible with China's third generation communication system - TD-SCDMA and the fourth FDD-LTE. With small size, high radiation intensity and wide operating bandwidth, this antenna can be put into mobile communication terminals.

In order to realize the aim of design, we combined microstrip antenna, cage dipole antenna and circular fractal antenna, these three technologies together. In order to solve the miniaturization problem of the cage dipole antenna first, we combined microstrip antenna and cage dipole antenna to design a microstrip cage dipole antenna, it obtains the excellent performance of these two antennas at the same time. Micropstrip cage dipole antenna can operate in a wide-band condition, but due to the exceeding horizontal branches in the middle of dipole arm, radiofrequency current would be over dispersed and the radiation intensity would decrease. In order to increase the radiation performance of antenna, we substituted the horizontal branches with circular fractal structures, which has multiple circuits. Meanwhile, the outer circuit of this structure directly connects with the feeding points, so that it can generate feeding radiation like microstrip cage dipole, however, the inner circuit can partly absorb radiation from outer circuit and create induct radiation. Therefore, feeding radiation and induct radiation can have superposition, so that the radiation performance can be highly increased. The structure of the antenna is shown in Figure 2. 


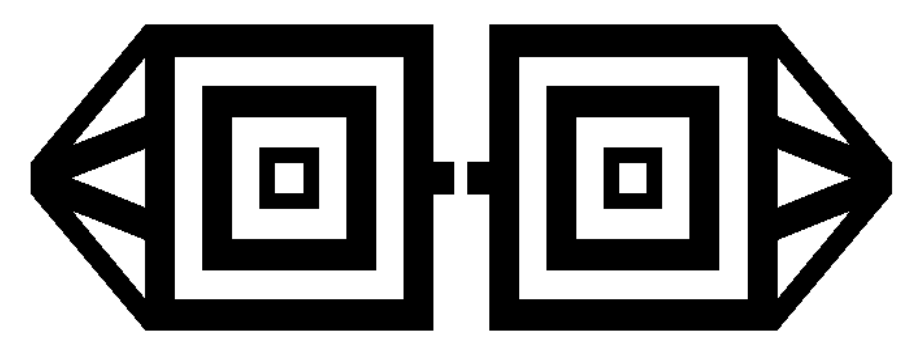

Fig.2. The scheme of the structure of wideband circular dipole antenna

The antenna includes the substrate and the dipole antenna radiation paster covered on the substrate, the latter of which is constituted by a pair of symmetrical dipole arms. Each dipole arm is composed by a feeding point, dual rectangular and fractal induced radiation structure, and end branch structure. The dual rectangular and fractal induced radiation structure on the dipole arm includes three levels of induced radiation rings which belong to the plane square metal ones. The end branch structure includes four radiation branches. The substrate used by the antenna is the FR4 dielectric substrate whose relative dielectric constant is 1.5. The shape is rectangle, and the antenna size is $30 \mathrm{~mm} \times 10 \mathrm{~mm} \times 2 \mathrm{~mm}$.

\section{The Simulation Analysis of Antenna's Performance}

We use the method of moments (MoM) to simulation analysis the performance of the antenna, the result of antenna's return loss performance shown in Figure 3.

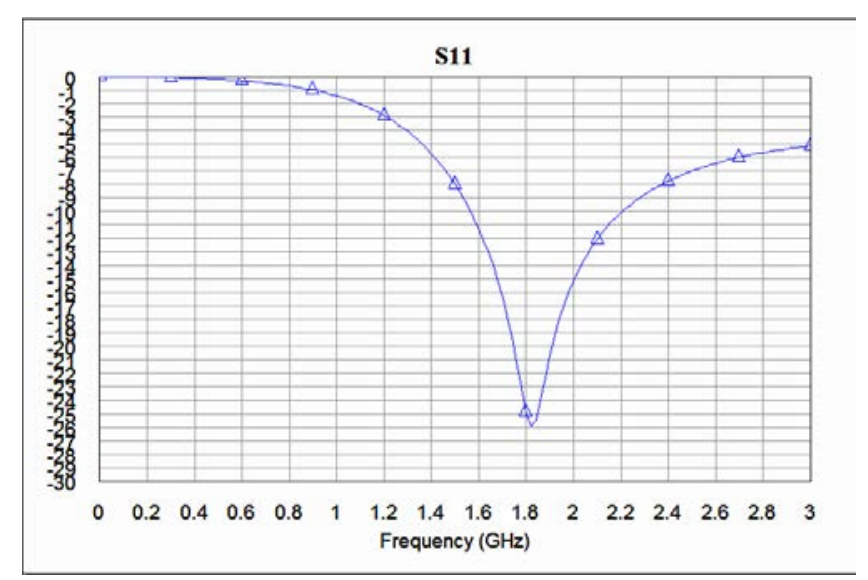

Fig.3. The simulation results of the antenna radiation characteristics

As shown in Figure 3, resonant point of the antenna was $1.82 \mathrm{GHz}$. The return loss were under $-10 \mathrm{~dB}$ in the whole working frequency band. The bandwidth reached $0.646 \mathrm{GHz}(1.562-2.208 \mathrm{GHz})$ and relative bandwidth was $37.27 \%$. This antenna fully covered the communication frequency of 1880-1920 MHz, 2010-2025 MHz of TD-SCDMA system and also the communication frequency of $1710-1785 \mathrm{MHz}, 1880-1920 \mathrm{MHz}$ of FDD-LTE system, realizing the design purpose.

\section{The Manufacture and Test of The Antenna Sample}

After the simulation analysis, we also made an antenna sample by etching process which as shown in Figure 4. 


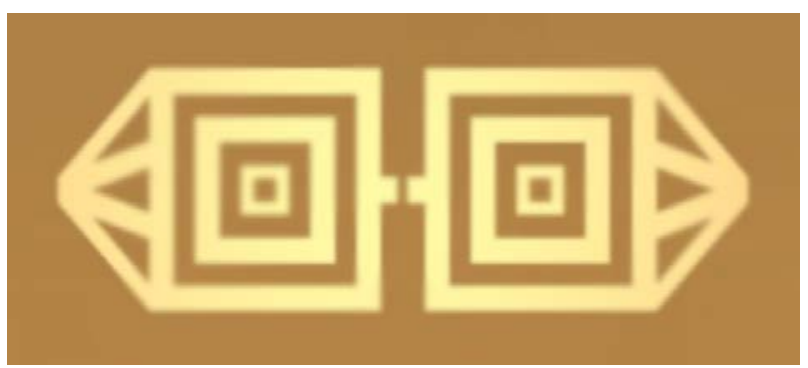

Fig.4. The photo of the antenna sample

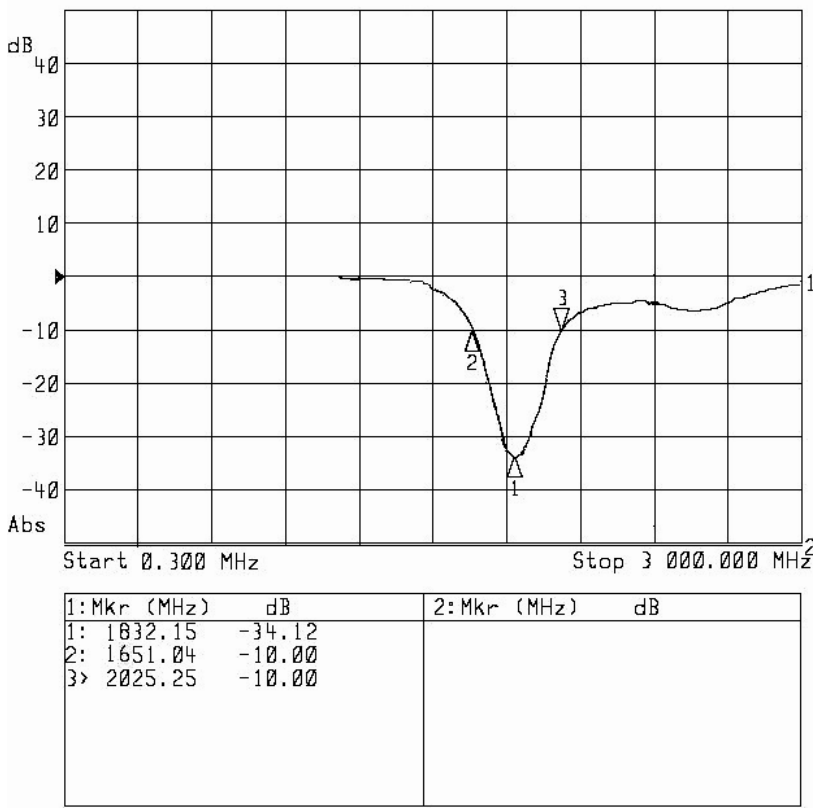

Fig.5. The experimental results of the antenna's return loss

We adopt RF integrated vector network analyzer to measure the return loss of the antenna, the results are shown in Figure.5. The results show that this antenna's resonant frequency is at the point of $1.832 \mathrm{GHz}$, the value of $\mathrm{S}_{11}$ return loss at resonant frequency is $34.12 \mathrm{~dB}$, while the operating frequency range is $1.651-2.025 \mathrm{GHz}$ and the bandwidth is $0.374 \mathrm{GHz}$.

Antenna is a high practical radio frequency apparatus. When we are designing the antenna, close results of simulative test and real test are required, thus comparing the results of both test are necessary. The comparison result of both tests (one from the simulative test of the improved wide-band circular dipole antenna (Firgure.3), the other one is from the real test (Figure.5)) shows a close result, but the simulative one is better than the real one. However, it is conforms to engineering reality. The minimal return loss of simulative antenna is $-25.91 \mathrm{~dB}$ with a bandwidth of $0.64 \mathrm{GHz}$, while the values of the return loss and operating bandwidth in the real test are smaller. This result caused by the designed software for the simulative test. The software default values are set to be the best condition for antenna operating. Therefore, antenna's radiofrequency current is in ideally distributed and radiation energy would be distribute on wider frequency band as far as possible, so the return loss would not be very small but the operating bandwidth would be big. However, in the real test, our building and welding process still exist some error, the real sample antenna does not fit the ideal one perfectly. Therefore, the radiofrequency current cannot be ideally distributed as simulative test. Although the sample antenna in the real test has approximately the same radiation power in the simulative test, the frequency range is smaller, thus it causes a smaller return loss and operating frequency band.

It is common to have little difference between real and simulative tests. There are two ways to solve this problem; one is improve the technology level, for example, use high accurate metal physical vapor deposition instead of corrosion technology, which has smaller error. The other one is that more precise structure can be designed to fit the antenna, such as a proper set of intelligent 
network at the back of the antenna.

\section{Conclusion}

This paper focuses on the requirements for wide frequency of antenna with the development of mobile communications. We managed to design a new wideband circular dipole antenna, which combines the three advantages of wideband microtrip antenna, circular fractal antenna and cage dipole antenna. Via the simulation analysis of the antenna's performance by moment method (MoM), we produced samples in use of testing. The simulation and experimental results show that this kind of antenna has strong radiation intensity, low return loss, wide working bandwidth. It can fully covered the communication frequency of $1880-1920 \mathrm{MHz}, 2010-2025 \mathrm{MHz}$ of the third generation mobile communication TD-SCDMA system and also the communication frequency of $1710-1785 \mathrm{MHz}, 1880-1920 \mathrm{MHz}$ of the fourth mobile communication FDD-LTE system, in another hand, realize the compatibility of the third and the fourth generation mobile communication.

This kind of antenna, whose size is only $30 \mathrm{~mm}$ by $10 \mathrm{~mm}$, realized miniaturization quiet well. It is smaller than human knuckle and its thickness is only $2 \mathrm{~mm}$. There is no doubt that this antenna can be put into mobile communication terminals, which has broad application foreground.

\section{References}

[1] Jiefeng Ao. The optimizing design of the PIFA based on the algorithm of orthogonality particle swarm [J]. Information Communication, 2015 (3) 67-68.

[2] Yulin Lei, Hui Zhang, Rongrong Zhang. Design of the application of wideband antenna of WLAN [J]. Modern Electronic Technology, 2011 (19) 87-90.

[3] Ying Liu, Shuxi Gong. The antenna in the mobile communication system. Beijing: Electronic Industry Press, 2011.

[4] Bekali Younes Karfa, Essaaidi Mohamed. Compact reconfigurable dual frequency microstrip patch antenna for 3G and 4G mobile communication technologies [J]. Microwave and Optical Technology Letters, 201355 (7) 1622-1626.

[5] Behera Subhrakanta, Vinoy K.J. Multi-port network approach for the analysis of dual band fractal microstrip antennas [J]. IEEE Transactions on Antennas and Propagation, 201260 (11) 5100-5106.

[6] Rani Shweta, Singh A.P. Modified Koch fractal antenna with asymmetrical ground plane for multi and UWB applications [J]. International Journal of Applied Electromagnetics and Mechanics, 201342 (2) 259-267.

[7] Zhigang Ye, Jingwan Wang. Process reform of HR4/2/0.5 cage oscillator of shortwave antenna [J]. Cable TV Technology, 2013 (11) 104-105. 